\section{IMMUNOTHERAPY}

\section{CARs for macrophages}

Nat. Biotechnol. https://doi.org/10.1038/s41587020-0462-y (2020)

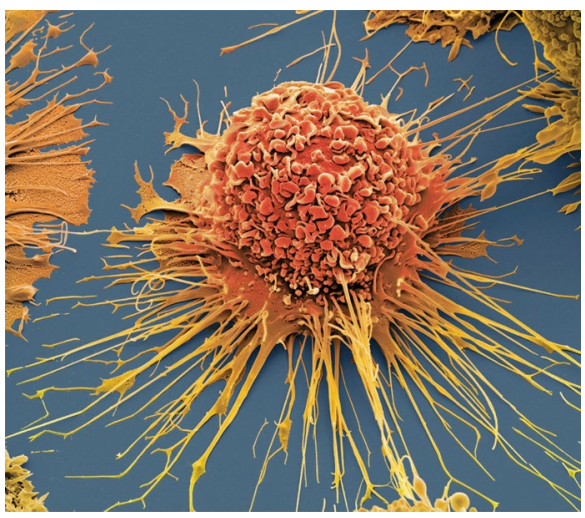

Credit: Science Photo Library/Alamy Stock Photo

Macrophages that express a chimeric antigen receptor (CAR) show promise for targeting solid tumors.

CAR T cells have been successful in targeting many blood cancers, but there has been limited success in treating solid cancers with them. It is thought that this is because $\mathrm{T}$ cells have difficulty in penetrating the tumor microenvironment. Macrophages are present in the tumor milieu, however, and could be useful to mount an initial anti-tumor response

Klichinsky et al. were able to transduce human macrophages with an adenovirus vector carrying a CAR. They show in humanized mouse models that infusion with this presumably resulted in a proinflammatory tumor microenvironment that activated $\mathrm{T}$ cells. The next stage will be a phase 1 trial to test safety in humans.

https://doi.org/10.1038/s41591-020-0905-5

\section{CELL THERAPY}

\section{Patching up hearts}

Sci. Transl. Med. 12, eaat9683 (2020)

An off-the-shelf synthetic cardiac patch promotes cardiac recovery in a rat model of acute myocardial infarction.

Myocardial infarction frequently leads to permanent arrhythmias and heart failure due to the remodeling process after these events. Cell therapy has been proposed as a means whereby more permanent damage could be avoided; however, it suffers from drawbacks such as cells being 'washed away' by the beating heart, immune incompatibility and high expense.

Huang et al. fabricated an artificial cardiac patch made of a porcine extracellular-matrix scaffold and synthetic cardiac stromal cells, which are encapsulated secreted human cardiac cellular factors. The patch withstood cryopreservation and supported cardiac recovery in a rat model of myocardial infarction. The cell-based patch thus shows promise for clinical translation.

\title{
CANCER THERAPY
}

\section{Precision-medicine success in pancreatic cancer

Matching people with pancreatic cancer to relevant molecularly guided treatments increases the overall survival of patients relative to that of those without actionable mutations or those who do not receive targeted therapy.

One of the goals of characterizing cancer mutations is to identify therapies that target those mutations and reduce cancer growth. However, there are limited real-world examples of the benefits of this.

The Know Your Tumor program recruited 1,856 US patients with pancreatic cancer; within this cohort, 46 had actionable mutations and were given relevant tailored therapies. Their median survival was significantly longer than that of both those with actionable mutations who were not given tailored therapies and those without actionable mutations. These real-world data suggest promise for this personalized approach.

\section{GENETIC DISORDERS}

\section{A therapy for $\boldsymbol{\beta}$-thalassemia \\ N. Engl. J. Med. 382, 1219-1231 (2020)}

HS Treatment of people with transfusiondependent $\beta$-thalassemia with luspatercept in a phase 3 trial resulted in a reduction in transfusion burden.

The $\beta$-thalassemias are a set of inherited hemoglobin disorders that result in an imbalanced ratio of globin chains and, hence, chronic anemia. In one subset of these disorders, the affected people need regular transplants to maintain hemoglobin levels, which is burdensome and exposes these people to infection. Luspatercept is a recombinant fusion protein that enhances blood-cell maturation and has shown promise in treatment for transfusion-dependent $\beta$-thalassemia.

Capellini et al. carried out a phase 3 trial of 224 patients given luspatercept and 112 patients given a placebo. They found that this treatment resulted in a significant reduction in transfusion burden for those treated and is suitable for clinical use. HS

https://doi.org/10.1038/s41591-020-0906-4

\section{PSYCHIATRIC DISORDERS \\ Predicting the first episode of psychosis}

Lancet Digital Health https://doi.org/10.1016/ S2589-7500(20)30024-8 (2020)

Machine learning can be used to predict the first episode of psychosis in people with schizophrenia 1 year before its occurrence.

Schizophrenia is a disabling psychiatric disorder, and the first episode of psychosis defines long-term outcomes. There are interventions that can prevent this event and could improve outcomes if used at the right time.

Raket et al. used a complied set of data from diverse electronic health record databases to develop the DETECT ('dynamic electronic health record detection') neural network to predict the first event of psychosis in people with schizophrenia. The model should be refined in further population-based settings, but they found it had adequate prediction accuracy.

https://doi.org/10.1038/s41591-020-0907-3

Hannah Stower 